\title{
DESENVOLVIMENTO HUMANO E ACESSO A SERVIC̣OS: UMA ANÁLISE ESPACIAL PARA OS MUNICIPIOS BRASILEIROS
}

\author{
Georgia Fernandes Barros ${ }^{1}$ \\ Ana Maria Hermeto Camilo de Oliveira ${ }^{2}$ \\ Pedro Vasconcelos Maia do Amaral ${ }^{3}$
}

\begin{abstract}
Resumo
O trabalho objetiva estudar teórica e empiricamente a mensuração e a distribuição do acesso a serviços e sua relação com o desenvolvimento humano nos municípios brasileiros nos anos 2000 e 2010, a partir de uma perspectiva multidimensional e espacial. A hipótese do trabalho é a de que existem padrões espaciais quando se relaciona o acesso a serviços e o desenvolvimento humano nos municípios. Em termos metodológicos, é construído um índice multidimensional de acesso a serviços e realizada uma análise do relacionamento desse índice com o IDHm nos municípios brasileiros. Os resultados apontam para uma conformação espacial desigual desses índices, tanto em termos regionais, quanto em termos de tamanho e hierarquia de municípios, indicando um entrelaçamento maior em locais tradicionalmente mais desenvolvidos, embora se observem melhorias pronunciadas na qualidade de vida nas regióes Nordeste e Norte do país e em municípios menos adensados e diversificados.
\end{abstract}

Palavras- chave: Desenvolvimento; Serviços; Multidimensionalidade; Análise espacial.

\section{HUMAN DEVELOPMENT AND ACCESS TO SERVICES: A SPATIAL ANALYSIS FOR BRAZILIAN MUNICIPALITIES}

\begin{abstract}
The work aims to study theoretically and empirically measure and the distribution of access to services and its relationship to human development in Brazilian cities in 2000 and 2010, from a multidimensional perspective and space. The hypothesis of this study is that there are spatial patterns as it relates access to services and human development in the municipalities. In terms of methodology, a multidimensional index of access to services is built and held one relationship analysis of this index with IDHM in Brazilian municipalities. The results point to an uneven spatial conformation of these indices, both regionally and in terms of size and hierarchy of cities, indicating a greater entanglement in places traditionally more developed, although there was pronounced
\end{abstract}

Recebimento: $12 / 7 / 2017 \cdot$ Aceite: $25 / 9 / 2018$

${ }^{1}$ Doutora em Economia Regional pelo Programa de Pós-Graduação em Economia do CEDEPLAR/UFMG. Professora da Universidade Federal dos Vales do Jequitinhonha e Mucuri, Teofilo Otoni - MG, Brasil. E-mail: georgiafbarros@gmail.com ${ }^{2}$ Doutora em Demografia pela Universidade Federal de Minas Gerais (UFMG). Professora do Departamento de Ciências Econômicas da UFMG, Belo Horizonte - MG, Brasil. E-mail: aherrmeto@cedeplar.ufmg.br

${ }^{3}$ Ph.D. em Land Economy pela University of Cambridge. Professor da Faculdade de Ciências Econômicas da UFMG e Fellow do Center for Spatial Data Science (University of Chicago), Belo Horizonte - MG, Brasil. E-mail: pedrovmm@gmail.com 
improvement in quality of life in Northeast and North regions the country and in less dense and diverse municipalities.

Keywords: Development; Services; Multidimensionality; Spatial analysis.

\section{Introdução}

Com a aceleração do processo de urbanização, a separação da população no espaço passa a ter impacto sobre a forma como as pessoas vivem e se reproduzem socialmente. Se por um lado, a urbanização trouxe benefícios relativos à maior facilidade de acesso ao progresso técnico/científico, às oportunidades laborais, educacionais, culturais, de infraestrutura etc., por outro lado, quando a análise se volta para alguns países da América Latina, como é o caso do Brasil, a concentração urbana ampliou as distâncias sociais, de maneira que as cidades, em especial as grandes cidades, tornaramse o lócus da desigualdade social e da concentração de pobreza.

A segregação sócio-espacial tem, assim, efeito significativo sobre a qualidade de vida das pessoas, de modo que as oportunidades presentes e usufruídas, além de serem determinadas por uma multiplicidade de fatores de ordem econômica, social e política, encontram-se relacionadas com o espaço. Ao se considerar a desigualdade de forma multifacetada, é possível conhecer de forma mais dinâmica e consistente o desenvolvimento assim como as suas especificidades locais e constitutivas, a partir de uma percepção mais complexa da realidade.

Nesse contexto, o presente trabalho tem como objetivo analisar as relações espaciais entre $o$ Índice de Desenvolvimento Humano municipal e um índice proposto de acesso a serviços nos anos 2000 e 2010, a partir de uma perspectiva espacial e multidimensional dessas relações. Parte-se do principio de que o desenvolvimento humano requer a inclusão dos indivíduos social, econômica e politicamente e que, portanto, faz-se necessário que as potencialidades das pessoas sejam incentivadas pelas instituições. A esse respeito, reitera-se a relevância do poder público local, consolidado através de maiores investimentos em saúde, educação, seguridade humana e expansão das capacidades e das oportunidades.

Em termos metodológicos, é utilizada a Análise Exploratória de Dados Espaciais - AEDE, para identificar a existência de clusters espaciais e a distribuição espacial das mudanças ocorridas no desenvolvimento dos municípios ao longo do período considerado e suas relações com a evolução do acesso a serviços.

O trabalho está dividido em 5 partes. Após essa Introdução, é apresentado o referencial teórico utilizado. No item 3, é apresentada a metodologia a ser utilizada, a Análise Exploratória de dados espaciais -AEDE. Os resultados são discutidos no item 4. Por fim, são apresentadas as conclusões do trabalho.

\section{Revisão de literatura}

O debate em torno da pobreza, da desigualdade, do baixo desenvolvimento e da exclusão social é recorrente nos campos da Filosofia, Economia e das Ciências Sociais. Desde o início deste século, entretanto, intensificaram-se as pesquisas em torno da complexidade desses fenômenos, em especial, sobre sua articulação em relação a diversos outros aspectos da realidade econômica e social contemporânea.

Com fundamento nessa literatura, abre-se espaço para o questionamento sobre o tamanho real da pobreza e da desigualdade e da amplitude do desenvolvimento, tendo em vista a necessidade de se considerar a pluralidade de questões envolvidas, como a expectativa de vida, a alfabetização, a liberdade, saúde, dentre outras. Por essa perspectiva, propõe-se uma visão de pobreza e de desigualdade baseada em uma perspectiva não unicamente monetária, ou seja, em uma perspectiva multidimensional.

Em grande medida respaldada nos trabalhos de Sen (1980, 1992, 2000, 2009), essa abordagem multidimensional conduz a um enfoque diferente daquele utilizado pela literatura tradicional da abordagem da pobreza e da desigualdade. A baixa renda é apenas um fator dos muitos que se exprimem pelas privações de capacidades. Desse modo, essa abordagem multidimensional da pobreza e da desigualdade apresenta uma concepção de desenvolvimento centrado no ser humano, em contraposição às análises tradicionais unidimensionais, em que o desenvolvimento está atrelado ao Produto Interno Bruto ou à renda per capita ou ao consumo. 
Para além do aumento da renda, o desenvolvimento implica a expansão das liberdades dos indivíduos em uma sociedade, sejam elas liberdade políticas, econômicas, sociais, de acesso a serviços, dentre outras ${ }^{4}$, de tal forma que um país será tanto mais desenvolvido quanto mais se promover a expansão do horizonte de liberdade dos seus cidadãos, o que significa que eles têm capacidades crescentes de ser e de fazer aquilo que valorizam e que têm razões para valorizar.

Em termos da multidimensionalidade da análise proposta, o movimento em direção a uma concepção mais complexa da pobreza e da desigualdade possui variadas contribuições, com referência nas obras de Rawls (1971), com sua abordagem da teoria de justiça distributiva, Sen (1980, 1992, 2000, 2009), que desenvolve as noções de funcionamentos e capacitações, Dworkin (1981), com a abordagem igualitária, e Roemer (1998), o qual aponta para a existência de fatores caracterizados pela responsabilidade dos indivíduos na formulação das normas de justiça social ${ }^{5}$.

A complexidade das relações entre renda e capacitações humanas possui implicações sobre as políticas públicas. As políticas de crescimento econômico devem ser analisadas não somente no que diz respeito ao seu impacto sobre a renda como também no que tange aos seus efeitos sobre as liberdades pessoais, o que irá depender da habilidade do Estado em oferecer serviços sociais estruturadores e capacitantes aos indivíduos. Desse modo, as políticas de crescimento e de ampliação das capacidades individuais devem se complementar mutuamente.

Em conformidade com Sen, o crescimento econômico possibilita não apenas no aumento da renda, mas pode permitir ao Estado financiar a seguridade social e a criação de oportunidades sociais como educação pública, serviços de saúde e desenvolvimento de uma imprensa livre e ativa pode contribuir para o desenvolvimento econômico e para uma redução significativa da taxa de natalidade (SEN, 2000, p. 61). O autor reitera, pois, a importância da conformação de governos democráticos, os quais permitem as pessoas desenvolverem seus funcionamentos, ampliarem as suas capacidades e assim levarem a vida que desejam.

Uma literatura recente tem tratado da multidimensionalidade no que tange ao acesso a serviços no espaço. Em âmbito nacional, citam-se os trabalhos de Picolotto (2006), que utilizou os funcionamentos saúde, nutrição, educação, política, solidariedade, liberdade e satisfação para analisar a pobreza e o desenvolvimento na região metropolitana de Porto Alegre, Assis (2012), que realiza uma revisão teórica sobre o contexto e as políticas de acesso a serviços de saúde, Carvalho, Kerstenetzky e Del Vecchio (2007), os quais buscam operacionalizar um conceito multidimensional, relativo e subjetivo da pobreza, utilizando indicadores de renda, educação, saneamento básico e condições domiciliares, população e saúde para as regiões metropolitanas da região sudeste do Brasil, Ottonelli e Mariano (2014), que tratam da pobreza multidimensional nos municípios do nordeste, utilizando as dimensões de saúde, educação, condições habitacionais e renda, dentre outros. Tais trabalhos têm em comum a busca pelas múltiplas faces do problema da pobreza, que englobam aspectos sociais, ambientais e econômicos.

No âmbito dos estudos sobre desenvolvimento humano, os Relatórios disponibilizados pelo Programa das Nações Unidas para o Desenvolvimento - PNUD têm ressaltado a importância do crescimento econômico local com ampliação da participação política nas esferas locais, com descentralização dos gastos e expansão da responsabilidade dos governos municipais e regionais e de uma administração pública voltada para os interesses coletivos como condição fundamental para o desenvolvimento. Apontam para a prioridade dos gastos públicos no que diz respeito à prestação de serviços sociais básicos, à geração de oportunidades e ao acesso a bens de produção. Em conformidade com esses trabalhos, é no âmbito local que se encontram as maiores possibilidades de efetivação de diversas práticas que poderiam impulsionar o desenvolvimento humano.

\footnotetext{
${ }^{4}$ Uma concepção adequada do desenvolvimento tem de ir muito além da acumulação de riqueza, do crescimento do produto nacional bruto (PNB) e de outras variáveis relacionadas à renda. Sem ignorar a importância do crescimento econômico, temos de olhar muito além dele (SEN, 2000, p.14).

${ }^{5}$ A multidimensionalidade da pobreza e da desigualdade, a partir dessas referências, foi discutida por Thorbecke (2002, 2008), Esposito e Chiappero-Martinetti (2008), Bourguignon e Chakravarty (2003), Bibi (2005), Bossert, Chakravarty e D'ambrosio (2009), Alkire e Santos (2010), Alkire e Foster (2011), Alkire et al. (2015). No âmbito dos estudos nacionais, podem ser citados os trabalhos de Hoffmann e Kageyama (2006), Barros, Carvalho e Franco (2006), Silva e Barros (2006), Silva e Neder (2010), Machado, Golgher e Antigo (2014), Bender Filho e Bagolin (2014), Bader et al. (2015), Pacheco, Del Vecchio e Kerstenetzky (2010), dentre outros.
} 
A partir das discussões propostas por essa literatura, é indispensável a existência de instituições que planejem o desenvolvimento, a partir de um sistema sinérgico e acessível aos cidadãos, que promova a qualidade de vida e reduza as assimetrias regionais. Para tanto, são importantes as políticas públicas, em especial as políticas de urbanização, uma vez que a maior parte da população vive nas cidades.

Nesse sentido, o presente trabalho se orienta a analisar as relações entre o desenvolvimento humano municipal e as instituições intervenientes, a partir da utilização do Índice de Desenvolvimento Humano Municipal (IDHm) e suas dimensões constitutivas e de um índice de acesso a serviços, cuja metodologia de proposição está descrita no próximo item.

\section{Aspectos metodológicos}

Em relação aos procedimentos de construção do índice e indicadores de acesso a serviços, estes tiveram como objetivo procurar aferir a diversidade e complexidade da realidade social e econômica dos municípios. Tal índice foi então proposto como um recurso para se medir e avaliar idiossincrasias e realidades municipais no que diz respeito a realização e acesso a serviços.

A partir da escolha das dimensões constitutivas do índice multidimensional de acesso a serviços, pautada na literatura, foram selecionados indicadores representativos de cada dimensão, os quais estivessem disponíveis para todos os municípios brasileiros nos anos de 2000 e $2010^{6}$.

Desse modo, com o intuito de construir os índices e variáveis a serem utilizados, foram consideradas as seguintes fontes de dados: Censo Demográfico (2000, 2010), Censo Escolar (2000, 2010), Pesquisa de Informações Básicas Municipais - MUNIC (2001, 2009), Sistema de Informação sobre Mortalidade (2000, 2010), dados do Cadastro de Registro Civil (2000, 2010), a base de dados Região de Influência das Cidades -REGIC (2007) e a base de dados do Atlas de Desenvolvimento Humano (2000, 2010). Para a montagem das bases, foi necessária a compatibilização no que se refere aos municipios existentes em 2000 e 2010 sendo considerada a conformação territorial do ano de 2000.

As diferentes escalas dos indicadores foram convertidas em uma única, de modo que variassem de zero a um. Uma vez assim expressos, cada indicador está contemplado no intervalo $(0,1)$. Se o indicador expressar um atributo positivo, o valor mínimo é utilizado como o pior/menor valor, e o valor máximo, como o melhor/maior valor. Se o indicador expressar um valor negativo, substitui-se o valor mínimo pelo maior/melhor valor e o valor máximo pelo pior/menor valor.

A aglutinação foi realizada utilizando-se a média dos indicadores existentes, de modo que, para cada dimensão, foi gerado um subíndice com valores compreendidos entre 0 e 1 . Posteriormente, esses subíndices foram agregados, por meio de média aritmética, no índice de acesso a serviços (Quadro 1).

As quatro dimensões utilizadas para a construção do índice de acesso a serviços refletem o pressuposto de que o Estado, em suas variadas formas de atuação pode aumentar ou reduzir a desigualdade de oportunidades municipais. Desse modo, pressupõe-se que a privação de liberdades também se vincula à carência de serviços públicos e de assistência social. A esse respeito, esse índice foi construído para se demonstrar como a presença desses serviços pode influenciar 0 desenvolvimento, minorando ou acentuando as heterogeneidades presentes nos municípios.

Com o objetivo de analisar a distribuição espacial dos índices municipais propostos, foi utilizada a Análise Exploratória de Dados Espaciais (Anselin, 1994, 2005). A utilização da metodologia de AEDE se justifica por permitir identificar se os índices associados a uma dada unidade espacial (município) estão relacionados aos observados em outras unidades espaciais. A magnitude da autocorrelação espacial entre as áreas (no caso, municípios), evidencia como os valores estão correlacionados no espaço (Anselin et al, 2002), global e localmente. Para tanto, foi utilizado o I de Moran local (LISA) para se verificar padrões locais de autocorrelação espacial. A estatística I de Moran positiva significa que existe uma autocorrelação positiva, ou seja, valores altos (baixos) tendem a estar localizados na vizinhança de valores altos (baixos). Se o valor for negativo, o inverso ocorre: valores altos estarão cercados de valores baixos, e vice-versa.

\footnotetext{
${ }^{6} \mathrm{Na}$ presença de indicadores alternativos representativos de uma mesma dimensão, foi selecionado o que apresentasse 0 maior coeficiente de variação, dado pela razão entre a média e o desvio-padrão dos resultados.
} 
As versões bivariadas das estatísticas de autocorrelação LISA, também, foram empregadas para captar a correlação espacial do acesso a serviços com o índice de desenvolvimento humano dos municípios. Essa estatística dá uma indicação do grau de associação linear, positiva ou negativa, entre o valor de uma variável em uma dada locação I e a média de outra variável nas locações vizinhas.

Tendo em vista a dimensão espacial, os índices são analisados por tamanho e por hierarquia dos municípios. Essa análise permite fornecer elementos para a compreensão da dinâmica e da evolução do desenvolvimento dado o processo brasileiro de dispersão urbana ${ }^{7}$. A análise relacionada a tamanho fundamenta-se na constatação de que a complexidade do fenômeno urbano aprofundouse com fortes rebatimentos nas configurações espaciais e nos problemas sociais vivenciados pela população em cidades de diferentes tamanhos e regiões do Brasil. Por sua vez, a hierarquia dos municípios é considerada em termos da influência de um município em seu entorno, com referência no estudo de Região de Influência das Cidades (IBGE, 2007).

\begin{tabular}{|c|c|}
\hline ensão & Descrição \\
\hline \multirow{5}{*}{ de Saú } & Profissionais de saúde por mil habitantes \\
\hline & Total de leitos privados por mil habitantes \\
\hline & Total de leitos públicos por mil habitantes \\
\hline & Total de estabelecimentos de saúde por mil habitantes \\
\hline & Cobertura de vacinação (\%) \\
\hline \multirow{3}{*}{$\begin{array}{lr} & \text { Edu } \\
\text { cação } & \mathrm{e} \\
\text { Cultura } & \end{array}$} & Número de escolas por mil habitantes \\
\hline & $\begin{array}{l}\text { Grupamento formado por acesso à internet, número de salas de aula, } \\
\text { número de computadores, existência de biblioteca quadra de esportes, } \\
\text { laboratório de ciências e laboratório de informática. Assume valor } 0 \text { para as } \\
\text { escolas com pior situação e } 1 \text { para as escolas em melhor situação. }\end{array}$ \\
\hline & $\begin{array}{l}\text { Grupamento composto pelas variáveis existência de biblioteca pública, } \\
\text { museus, estádios, teatro, cinema, clube, orquestra no município. Assume valor } \\
0 \text { para os grupamentos com menores valores e } 1 \text { para aqueles com melhores } \\
\text { valores }^{8}\end{array}$ \\
\hline \multirow{3}{*}{$\begin{array}{lr}\text { da } & \text { Ren } \\
\text { trabalho } & \text { e }\end{array}$} & Existência de programas municipais de trabalho e renda \\
\hline & $\begin{array}{l}\text { Proporção de elegíveis que recebem Bolsa Família no município } \\
\text { (Focalização de inclusão) }\end{array}$ \\
\hline & $\begin{array}{l}\text { Proporção de não elegíveis que recebem Bolsa Família no município } \\
\text { (vazamento) }\end{array}$ \\
\hline \multirow{9}{*}{$\begin{array}{l}\quad \text { Inst } \\
\text { rumentos } \\
\text { gestão } \\
\text { urbana }\end{array}$} & $\begin{array}{l}\text { Existência de conselho municipal na área de política urbana, que tenha } \\
\text { se reunido nos últimos doze meses }\end{array}$ \\
\hline & $\begin{array}{l}\text { Existência de conselho municipal na área de cultura, que tenha se } \\
\text { reunido nos últimos doze meses }\end{array}$ \\
\hline & $\begin{array}{l}\text { Existência de conselho municipal na área de habitação, que tenha se } \\
\text { reunido nos últimos doze meses }\end{array}$ \\
\hline & Existência de fundo municipal de habitação \\
\hline & Existência de programas habitacionais no município \\
\hline & Existência de guarda municipal \\
\hline & Efetivo guarda municipal por mil habitantes \\
\hline & Existência de conselho municipal de meio ambiente \\
\hline & Existência de fundo municipal de meio ambiente \\
\hline
\end{tabular}

Fonte: Elaboração Própria a partir de dados do Censo Demográfico, Registro Civil, Datasus, Censo Escolar, anos 2000 e 2010 e Munic, 2001 e 2009.

\section{Resultados e discussão}

\footnotetext{
${ }^{7}$ Ver Simões e Amaral (2011), Diniz (2002).

${ }^{8}$ Foi utilizado o método não hierárquico de grupamento, conhecido como $\mathrm{K}$ means clustering.
} 
O índice de acesso a serviços foi construído com referência nas áreas de saúde, educação e cultura, renda e trabalho e instrumentos de gestão urbana. O Gráfico 1 retrata a evolução desse índice.

Em geral, ocorreu uma elevação da oferta de serviços (IAS) entre os anos considerados. Como pode ser percebido, houve uma piora dos resultados relativos a Renda e Trabalho em função da redução da oferta de programas municipais de trabalho e renda entre 2000 e 2010. As maiores variações ocorreram nas regiões nordeste $(0.63)$, norte $(0.57)$ e sul $(0.36)$.

GRÁFICO 1 - Evolução do Índice de Acesso a serviços e de seus componentes, 2000 e 2010.

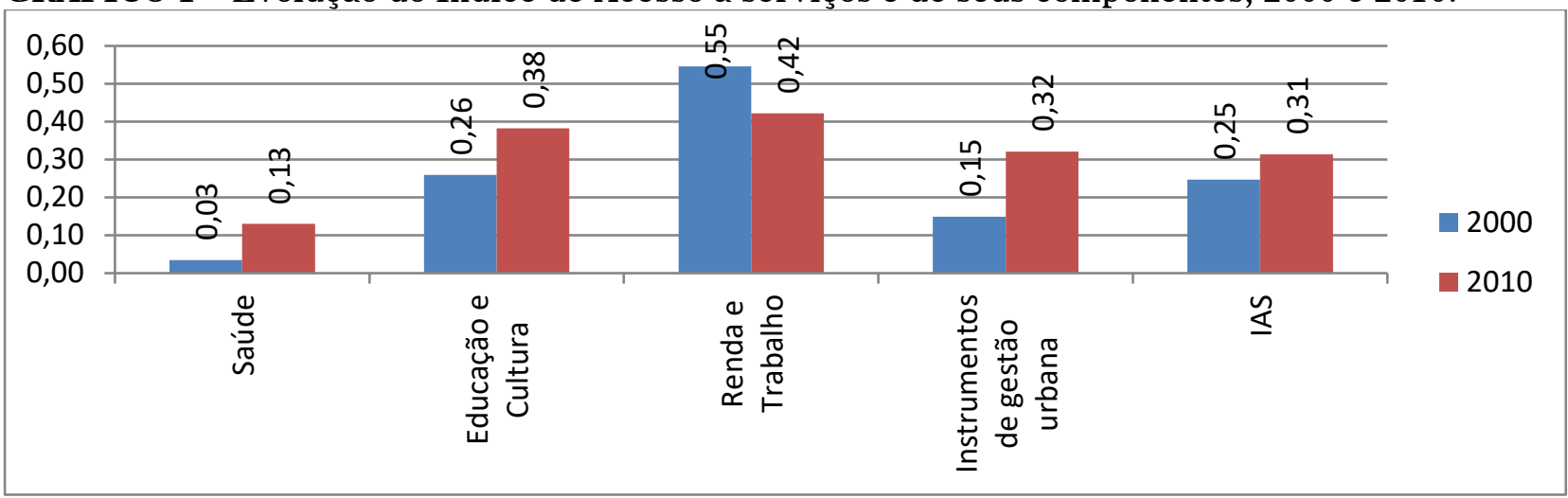

Fonte: Elaboração Própria a partir de dados do Censo Demográfico, Registro Civil, Datasus, Censo Escolar, anos 2000 e 2010 e Munic, 2001 e 2009.

A análise de autocorrelação espacial revela que nos anos 2000 e 2010, há uma maior concentração dos centros de grupamentos com maior acesso a serviços no sul (56,57\% em 2000 e $52,80 \%$ em 2010) e sudeste (32,37\% em 2000 e 27,24\% em 2010), indicando a existência de municípios com elevado valor desse índice de oferta circundados por municípios com elevados valores do referido índice e vice-versa (Figura 1). Esses desequilíbrios regionais se refletem em uma divisão de serviços hierarquizada a partir de centros urbanos hegemônicos que concentram as atividades mais complexas, a população e os principais mercados de bens e serviços, além de possuírem mercados de trabalho mais diversificados, de modo que, apesar dos avanços na oferta de serviços nos últimos anos, as desigualdades espaciais elucidam, sobretudo, as disparidades no nível de desenvolvimento social e econômico dos municípios.

Ao se considerar, entretanto, a autocorrelação espacial da variação do IAS entre esses anos considerados, é observado que ela é maior no nordeste $(75,4 \%)$ e em pontos da região norte $(11,25 \%)$. Desse modo, há um aumento da qualidade e do acesso a serviços, que embora permaneçam mais concentrados nas regiões mais desenvolvidas, apresentam um padrão de convergência em termos das regiões periféricas, em especial, destaca-se a região nordeste como aquela que apresentou maior crescimento desse índice.

Figura 1 - I de Moran Local para a variação do IAS

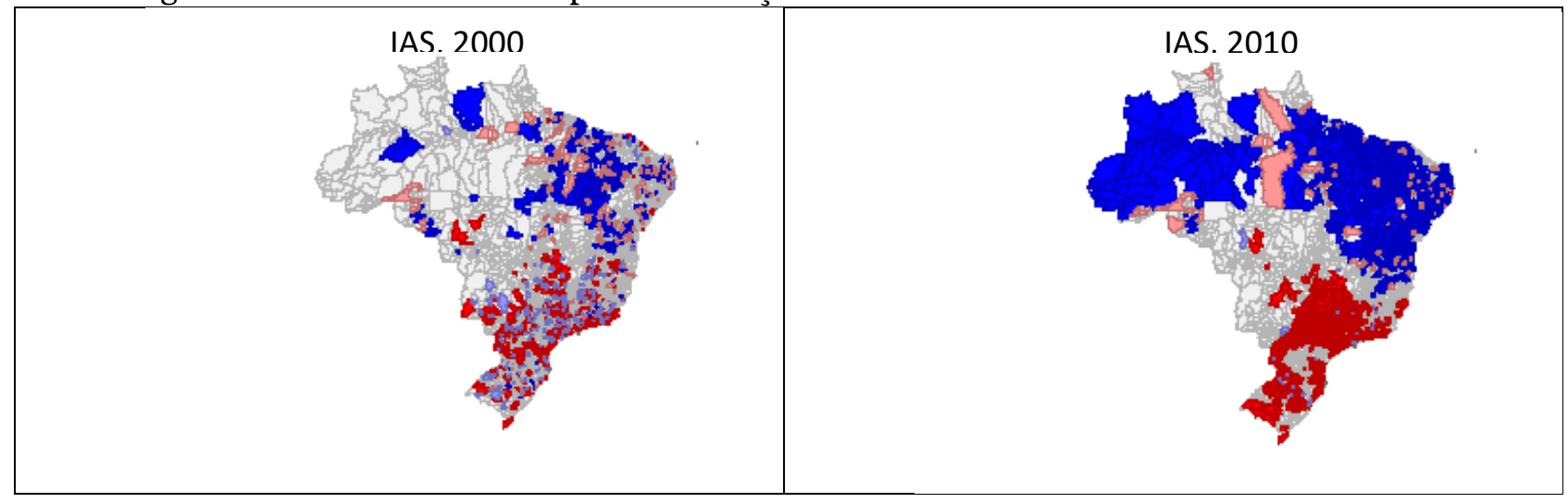




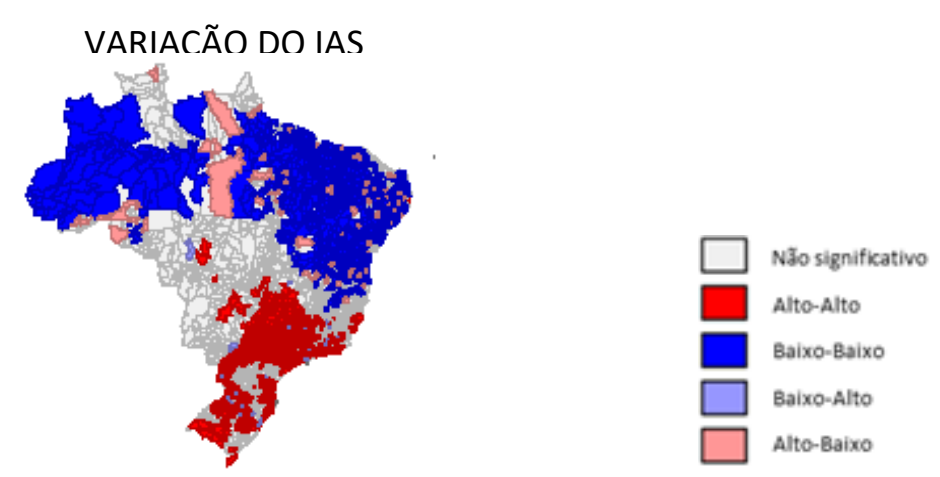

Fonte: Elaboração Própria a partir de dados do Censo Demográfico, Registro Civil, Datasus, Censo Escolar, anos 2000 e 2010 e Munic, 2001 e 2009.

Obs. Foi considerada uma matriz de vizinhança do tipo Queen de primeira ordem e um nível de significância de $5 \%$.

Na Figura 2, é retratada a distribuição espacial do IDHm nos anos 2000, 2010, assim como a variação do índice nesses anos. Observa-se uma grande desigualdade na distribuição desse índice, com padrão semelhante ao observado para a distribuição de serviços, com as regiões sul e sudeste concentrando os centros de cluster de maior valor de desenvolvimento humano e, desse modo, municípios com maiores oportunidades sociais e econômicas que possuem como vizinhos municípios em situação similar.

Já as regiões norte e nordeste concentram os piores valores desse índice, o que aponta para uma maior carência em termos de infraestrutura e oportunidades. Verifica-se também que há presença de autocorrelação espacial negativa, que se refere a altos valores de desenvolvimento em relação às baixas médias dos vizinhos, e vice-versa, caracterizando outiliers espaciais (clusters $\mathrm{AB}$ e BA).

Figura 2 - I de Moran local do IDHm e componentes, 2000, 2010 e variação

\begin{tabular}{|c|c|}
\hline IDHm & \\
\hline Variação do IDHm-2000 e & Variaça do IDHm, Longevidade-2000 \\
\hline & \\
\hline Variação do IDHm, Educação-2000 e & Variação do IDHm, Renda - 2000 e 2010 \\
\hline
\end{tabular}




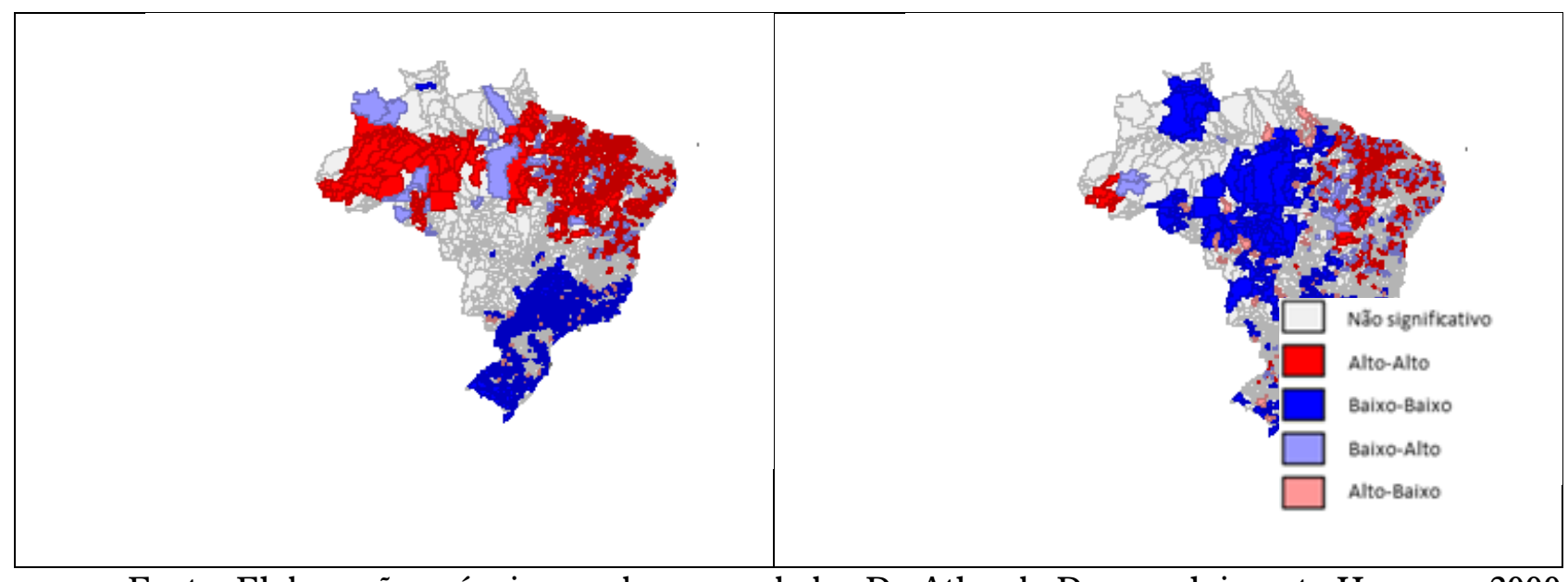
e 2010.

Fonte: Elaboração própria com base nos dados Do Atlas de Desenvolvimento Humano, 2000

Obs. Foi considerada uma matriz de vizinhança do tipo Queen de primeira ordem e um nível de significância de $5 \%$.

Como pode ser observado, os padrões de distribuição espacial de desenvolvimento municipal se assemelham ao padrão de distribuição dos serviços. Para se verificar a autocorrelação espacial local entre as variações do IAS e o IDHm e componentes, foi utilizado I de Moran bivariado (Figura $3)$. e 2010

Figura 3 - Coeficientes de I de Moran bivariado para as variações do IDHm e IAS entre 2000

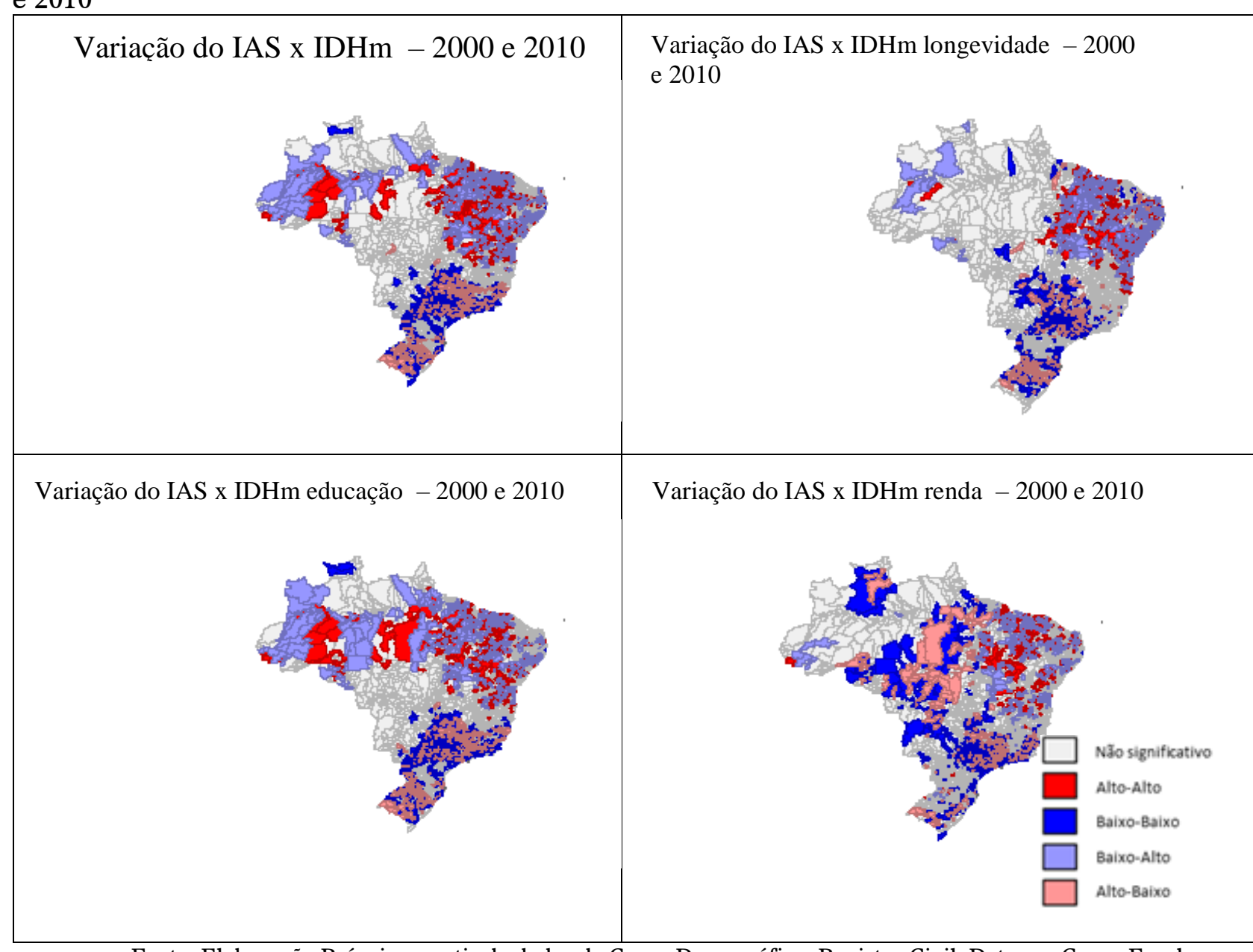

Fonte: Elaboração Própria a partir de dados do Censo Demográfico, Registro Civil, Datasus, Censo Escolar, anos 2000 e 2010 e Munic, 2001 e 2009, Atlas de Desenvolvimento Humano, 2000 e 2010. Obs. Foi considerada uma matriz de vizinhança do tipo Queen de primeira ordem e um nível de significância de 
De acordo com a Figura 3, o crescimento de aglomerações de municípios com melhores condições de oferta de serviços que possuem como vizinhos municípios com elevado IDHm (clusters Alto-Alto) se concentram nas regiões norte e nordeste. Análise semelhante pode ser feita em relação ao componente da educação do IDHm.

Para os componentes longevidade e renda, a análise bivariada da correlação espacial local identifica principalmente a região nordeste como agregadora dos clusters tipo Alto-Alto. Assim, os clusters de elevado crescimento do IDHm se encontram localizados nas regiões norte e nordeste, em que municípios que apresentam elevado padrão de variação de acesso a serviços são rodeados por municípios com elevada variação desenvolvimento humano. As regiões sul, sudeste e centro-oeste apresentam padrões de relação espacial entre desenvolvimento e serviços mais baixos. Os grupamentos de municípios com padrões Baixo-Alto e Alto-Baixo podem ser vistos como localidades em que não há o mesmo processo de dependência espacial das demais observações. Desse modo, os indicadores LISA sugerem que há uma dependência espacial entre a melhoria do desenvolvimento humano e do acesso a serviços nos municípios brasileiros.

Tendo em vista a heterogeneidade espacial do Brasil e as especificidades locais em termos de desenvolvimento, foi feito um estudo contemplando a relação entre os centros urbanos e as oportunidades existentes nos municípios. A esse respeito, as Figuras 4 e 5 apresentam, respectivamente, como IDHm (e componentes) se relacionam espacialmente com o IAS considerando o tamanho dos municípios e a sua hierarquia. De modo geral, as maiores variações na distribuição espacial dessas relações ocorrem nos municípios menos adensados e diversificados, com população entre 10.000 a 50.000 habitantes e nos municípios classificados como centros locais, centros de zona e centros sub-regionais.

Figura 4: Evolução do desempenho do IDHm e IAS por classes de tamanho da população

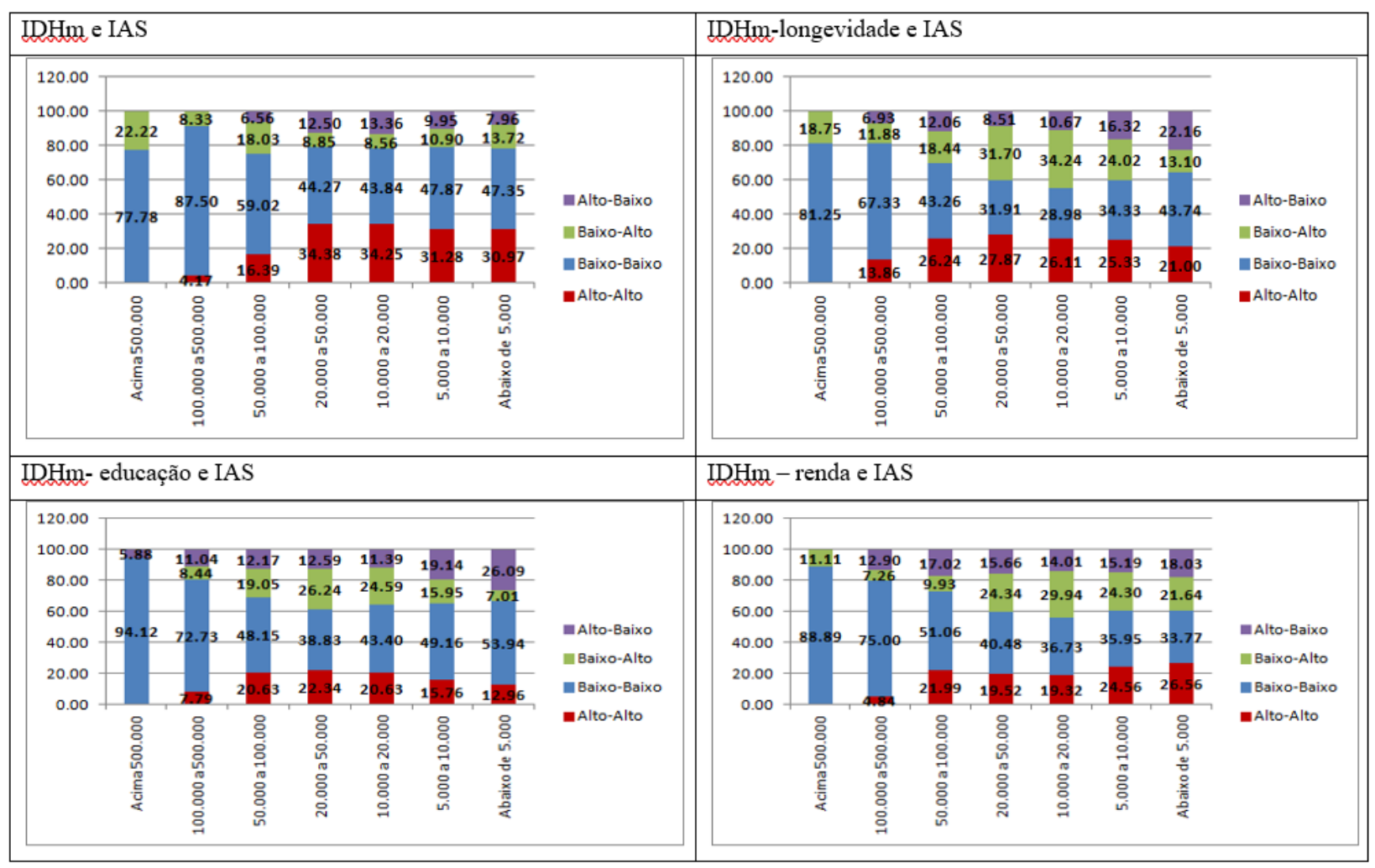

Fonte: Elaboração Própria a partir de dados do Censo Demográfico, Registro Civil, Datasus, Censo Escolar, anos 2000 e 2010 e Munic, 2001 e 2009, Atlas de Desenvolvimento Humano, 2000 e 2010. 
Figura 5: Evolução do desempenho do IDHm e IAS por hierarquia de municípios

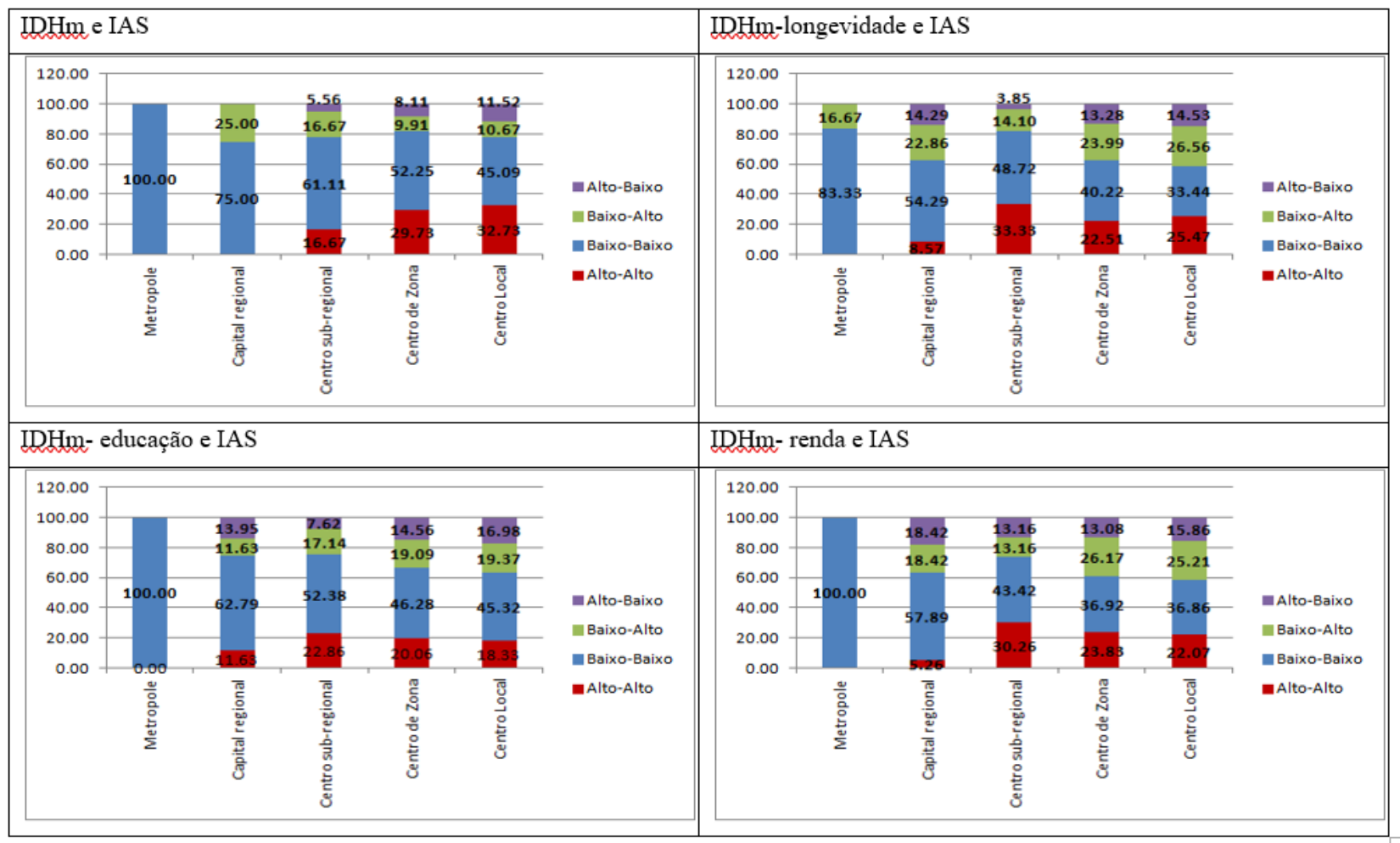

Fonte: Elaboração Própria a partir de dados do Censo Demográfico, Registro Civil, Datasus, Censo Escolar, anos 2000 e 2010 e Munic, 2001 e 2009, Atlas de Desenvolvimento Humano, 2000 e 2010.

\section{Conclusões}

Este trabalho teve como objetivo analisar espacialmente a evolução do acesso a serviços e sua relação com a evolução do desenvolvimento nos municípios brasileiros. Para tanto, foi proposto um índice multidimensional de serviços no intuito de se obter uma compreensão mais ampla sobre as mudanças estruturais no âmbito municipal, as quais potencializam o exercício dos direitos humanos e minoram a vulnerabilidade. Além disso, foi realizada uma análise espacial acerca de suas relações com o IDHm. A análise contemplou, assim, a relevância da concepção multidimensional e também da dimensão espacial, o que possibilitou uma compreensão mais ampla da dinâmica do desenvolvimento e de suas relações com os aspectos institucionais.

Em termos dos resultados obtidos, pôde ser observada uma melhoria nos índices no período considerando, indicando um maior desenvolvimento humano e também maior acesso a serviços nos municípios, embora persista uma conformação espacial desigual desses índices, tanto em termos regionais, quanto em termos de tamanho e hierarquia de municípios, indicando um entrelaçamento maior em locais tradicionalmente mais desenvolvidos. A despeito disso, foram percebidas melhorias pronunciadas na qualidade de vida nas regióes Nordeste e Norte do país e em municípios menos adensados e diversificados. Foi assim verificada uma estreita relação entre o IDHm e as condições de planejamento e desenvolvimento urbano ao longo do espaço brasileiro, o que permitiu aceitar a hipótese desse trabalho. Desse modo, as melhorias de condições de vida se relacionam a melhorias no acesso a serviços no espaço brasileiro.

Percebe-se, portanto, um processo de reestruturação sócio-espacial, com maior crescimento do IDHm e do IAS em regióes que apresentam historicamente índices de desenvolvimento socioeconômico relativamente piores, o que aponta para a existência de um sistema multifacetado de núcleos e periferias em inter-relação e hierarquia que caracterizam o espaço brasileiro e que não se apresenta estaticamente, ao contrário, caracteriza-se pela dinâmica e evolução das interações entre os municípios e regiões. 
Possíveis explicações para estes resultados são o arrefecimento do processo de metropolização brasileiro e movimento de interiorização da atividade econômica, a crescente importância que os municípios vêm assumindo em termos de direcionamento de políticas públicas e alocação de recursos em favor de segmentos ou grupos considerados prioritários, maior focalização da ação governamental em termos de programas sociais, com ênfase para o Programa Bolsa Família, o aumento da oferta e do acesso à qualificação profissional, ao ensino superior, dentre outras.

\section{Referências Bibliográficas}

ALKIRE, Sabina; FOSTER, James. Counting and multidimensional poverty measurement. Journal of Public Economics, v. 95, p. 476-487, 2011.

ALKIRE, Sabina; FOSTER, James; SETH, Suman; SANTOS, Maria Emma; ROCHE, José Manuel; BALLON, Paola. Multidimensional poverty measurement and analysis. Oxford: Oxford University Press, 2015.

ALKIRE, Sabina; SANTOS, Maria Emma. Acute multidimensional poverty: a new index for developing countries. OPHI Working Paper 38, University of Oxford, 2010.

ANSELIN, L. Exploratory spatial data analysis and geographic information systems. In: PAINHO, M. (Ed.) New tools for spatial analysis: proceedings of the workshop. Luxemburgo: EuroStat, 1994. p. $45-54$.

ANSELIN, L. Exploring Spatial Data with GeoDaTM: a Workbook. University of Illinois, UrbanaChampaign, 2005.

ANSELIN, L., I. SYABRI, O. SMIRNOV \& REN, Y. Visualizing Spatial Autocorrelation with Dynamically Linked Windows. Computing Science and Statistics, v. 33, 2002.

ASSIS, Marcos A. Acesso, cobertura e progressão escolar em Minas Gerais: que desigualdades? In: FAHEL, Murilo; RAMBLA, Xavier; LAZZAROTTI, Bruno; BRONZO, Carla (Org.). Desigualdades Educacionais e Pobreza. Belo Horizonte: PUC Minas, 2012.

BADER, C. et al. A different perspective on poverty in Lao PDR: Multidimensional poverty in Lao PDR for the years 2002/2003 and 2007/2008. Social Indicators Research, p. 1-20, 2015

BARROS, R. P.; CARVALHO, M.; FRANCO, S. Pobreza multidimensional no Brasil. Texto para discussão n. 1227. Rio de Janeiro: IPEA, out., 2006.

BENDER FILHO, R. ; BAGOLIN, I. P. Determinantes da permanência na condição de pobreza crônica na Cidade de Porto Alegre: aplicação do Modelo Logit Multinomial. Ensaios FEE, v. 35, n. 2, 2014.

BIBI, S. Measuring Poverty in a Multidimensional Perspective: A Review of Literature. PMMA Working Paper n. 2005-07, novembro 2005

BOSSERT, W.; CHAKRAVARTY, S. R.; D'AMBROSIO, C. Multidimensional Poverty and Material Deprivation. CIREQ-Cahier 12-2009, Montreal, ago. 2009

BOURGUIGNON, F.; CHAKRVART S. The Measurement of Multidimensional Poverty. The Journal of Economic Inequality, v.1, p. 25-49, 2003.

CARVALHO, M.; KERSTENETZKY, C. L.; DEL VECCHIO, R. Uma aplicação da teoria dos conjuntos fuzzy na pobreza: o casa das Regióes Metropolitanas do Sudeste brasileiro - 2000. In: Encontro Nacional de Economia (ANPEC), 35, 2007, Recife. Anais eletrônicos... Recife: Encontro Nacional de Economia, 2007. Disponível em: <http://www.anpec.org.br/encontro2007/artigos/A07A001.pdf>. Acesso em: 30 nov. 2015.

CHIAPPERO-MARTINETTI, E. A multidimensional assessment of well-being based on sen's functioning approach. Review Internationally of Science Socially, n. 2, p. 207-239, 2000.

DINIZ, C. C. A nova configuração urbano-industrial no Brasil. In: KON, A. (Ed.). Unidade e Fragmentação: A Questão Regional no Brasil São Paulo: Perspectiva, p. 87-115. 2002.

DWORKIN, R. What is Equality? Part 1: Equality of Welfare; Part 2: Equality of Resources., Philos. Public Affairs, v.10, p.185-246; 283-345, 1981. 
ESPOSITO, L.; CHIAPPERO-MARTINETTI, E. Multidimensional Poverty Measurement: Restricted and Unrestricted Hierarchi among Poverty Dimensions. OPHI Working Paper 22, Oxford, Agosto 2008

HOFFMANN, R.; KAGEYAMA, A. Pobreza no Brasil: uma perspectiva multidimensional. Revista Economia e Sociedade, Campinas, v.15, n.1 (26), p. 79-112, 2006.

MACHADO, A. F.; GOLGHER, A, B.; ANTIGO, M. F. La privación desde un enfoque multidimensional: el caso brasileño, Revista de la CEPAL, n.112, p. 129-150, 2014.

MARTINEZ JR, Arturo, Perales, F. The Dynamics of Multidimensional Poverty in Contemporary Australia. Social Indicators Research 130: 479-496, 2017.

MARTIROSOVA, Diana; INAN, Osman Kaan; MEYER, Moritz; Sinha, Nistha. . The Many Faces of Deprivation : A Multidimensional Approach to Poverty in Armenia. Policy Research Working Paper; n. 8179. World Bank, Washington, DC. (C) World Bank, 2017.

MITRA, Sophie, Debra L. BRUCKER. Income Poverty and Multiple Deprivations in a HighIncome Country: The Case of the United States. Social Science Quarterly, 2016.

OTTONELLI, J.; MARIANO, J. L.. Pobreza multidimensional nos municípios do nordeste. Revista Administração Pública, v. 48, n. 5, p. 1253-1279, set./out. 2014.

OTTONELLI, J. Pobreza Multidimensional na região Nordeste: uma aplicação da teoria dos conjuntos Fuzzy (em 2010). Dissertação (Mestrado em Economia) - Universidade Federal do Rio Grande do Norte, Natal, 2013.

PACHECO, K.; DEL-VECCHIO, R.; KERSTENETZKY, C. L. Pobreza Fuzzy multidimensional: uma análise das condições de vida na Zona Oeste do Rio de Janeiro: 1991 a 2000. Centro de Estudos sobre Desigualdade e Desenvolvimento (CEDE), Niterói. Texto para discussão n. 30, set. 2010.

PICOLOTTO, V. C. Pobreza e desenvolvimento sob os paradigmas da renda e das capacitações: uma aplicação para a Grande Porto Alegre através de indicadores fuzzy. Dissertação (Mestrado em Economia) - Universidade Federal do Rio Grande do Sul, Porto Alegre, 2006

RAVALLION, Martin. On Multidimensional Indices of Poverty”. Policy Research Working Paper 5580. The World Bank Development Research Group, 2011.

RAWLS, John. A Theory of Justice. Cambridge, Mass., Harvard University Press, 1971.

ROEMER, John E. Equality of Opportunity. Cambridge, MA: Harvard University Press, 1998.

SEN, A. Desenvolvimento como liberdade. São Paulo: Companhia das Letras, 2000.

SEN, A. Inequality Reexamined. Cambridge, Mass., Harvard University Press, 1992.

SEN, A. Equality of What? In: MCMURRIN, S. (org.). Tanner Lectures on Human Values. Cambridge, Cambridge University Press, 1980.

SEN, A. The Idea of Justice. London: Allen Lane, 2009.

SILVA, A. M. R.; NEDER, H. D. Abordagem das capacitações: um estudo empírico sobre pobreza multidimensional no Brasil. III Conferência Latino Americana e caribenha sobre Abordagem das Capacitações e Desenvolvimento Humano, Porto Alegre, 2010.

SILVA, M. C. P.; BARROS, R. P. Pobreza Multidimensional no Brasil. Anais... XXXIV Encontro Nacional de Economia 141, ANPEC - Associação Nacional dos Centros de Pós-graduação em Economia, 2006.

SIMÕES, R.; AMARAL, P. V M. do. Interiorização e Novas Centralidades Urbanas: Uma Visão prospectiva para o Brasil. Economia, ANPEC - Associação Nacional dos Centros de Pósgraduação em Economia [Brazilian Association of Graduate Programs in Economics], v. 12, n.3, p. 553-579, 2011.

THORBECKE, E. Multidimensional Poverty: Conceptual and Measurement Issues: Many Dimensions of Poverty, p.3-19, 2008. TSUI, K. Multidimensional poverty indices. Social Choice and Welfare, vol. 19, No 19. Spring, 2002. 
THORBECKE, E. Multidimensional Poverty: Conceptual and Measurement Issues: Many Dimensions of Poverty, p.3-19, 2008.

WHELAN, Christopher T., NOLAN, Brien, MAITRE, Bertrand. Multidimensional poverty measurement in Europe: An application of the adjusted headcount approach". Journal of European Social Policy, vol. 24, p. 183-197, 2014. 